\title{
Implementation of IEEE802.11 (Wi-Fi) in NS-3
}

\author{
Eliud Momanyi Manyinsa \\ Telecommunication and Information Engineering, Jomo Kenyatta University of Agriculture and Technology, Nairobi 00100, Kenya
}

\begin{abstract}
Computer networks technology is subject to constant change and innovation. New ideas and concepts regarding the usage of internet networks demand new network protocol and technologies. Due to its complexity many challenges arise from developing, implementing, testing and understanding these technologies. This is where simulation comes into play. Network Simulator is used to evaluate the performance of different wired and wireless networks and their topologies without real world implementation. Network Simulators are widely used by the research community to evaluate new theories and hypothesis. NS-3(network simulator 3) has a number of helper objects that assist in execution and analysis of simulation. The main focus of this paper is to study the Wi-Fi (wireless fidelity) performance when implemented on NS-3. This paper is offering a closer look on network simulation by describing NS-3, its core functionality and Wi-Fi implementation in NS-3.
\end{abstract}

Key words: Wireless networks, Wi-Fi, simulation, network simulators, NS-3.

\section{Introduction}

A wireless network is a type of computer network system which uses wireless connection for data transfer between different network nodes. The main reason of using wireless networks over wired networks is that it avoids installations of cables into a building which is a very complex and costly process to employ. It is for this reason that wireless network is growing in popularity arising from its mobility, simplicity, affordability and cost saving installation [1]. The rest of the paper is organized as follows: Section 1 continues with the explanation of types of wireless network and Wi-Fi(wireless fidelity); Section 2 introduces to the type of wireless network simulator and their comparisons mainly open source wireless network simulator; Section 3 discusses more about NS-3 (network simulator 3) and its organizational structure; Section 4 describes the Wi-Fi network simulation setup and discusses the results; The paper finally concludes the research in Section 5.

\subsection{Types of Wireless Networks}

A wireless network is a network setup using radio

Corresponding author: Eliud Momanyi Manyinsa, M.Sc., research field: telecommunication. signal frequency to communicate among computers and other network devices. A radio communication is the medium that connects devices to one another [2]. Wireless Networks are divided into two main groups which are infrastructure wireless network also known as centralized networks and Ad-Hoc or infrastructure less network.

\subsubsection{Infrastructure Network}

Infrastructure network is a fixed network that has base stations or access points from which wireless nodes can get connected. All the base stations or access points are connected with the main network through wired links or wireless links. The base station or access point is the important units on infrastructure networks. All connections pass to and from the access point where a wireless node can connect to any access points within its reach range.

\subsubsection{Ad-Hoc Network}

Ad-Hoc networks do not have infrastructure hence the nodes are free to join and leave the networks. The nodes connect with each other through a wireless link. A node may serve as a router to forward data to the neighbours' nodes. This type of networks is also termed as infrastructure less networks since it has no centralized administration [3]. Ad-Hoc network can handle any malfunction in the nodes or any changes 
that are experience due to topology changes. Whenever a node in the network is down or leaves the network, it causes the link with other nodes to be broken [4]. The affected nodes in the network simply request for new routes and new links are established. Ad-Hoc network can be categorised into STANET (static Ad-Hoc network) and MANET (mobile Ad-Hoc network) [5].

\subsection{Wi-Fi}

Wi-Fi is based on the IEEE standard 802.11. It operates on $2.4 \mathrm{GHz}$ and $5 \mathrm{GHz}$ frequency band and provides data transfer at maximum rate of 54 Mbps. Wi-Fi has two types of components: a wireless client station and an AP (access point). A wireless client station can be any user device such as computer or laptop that has a wireless network card. AP acts as a bridge between fixed and wireless networks. It connects to the cable modem or DSL (digital subscriber line) modem to allow internet services to the wireless and wired clients. Users can connect smart phones to wireless routers for Wi-Fi services and access the internet over mobile phone wireless [6].

\section{Wireless Network Simulators}

Simulation is a technique where a program models the behaviour of network either by calculating the interaction between the different network entities using mathematical formulas or actually capturing and playing back observations from production network. The network simulator is software that predicts behaviour of the computer network which is modelled with devices, links, applications and the performance is analysed [7]. Users can then customize the simulator according to their specific analysis needs. Simulators come with support for the most popular protocols and networks in use today such as WLAN, WiMAX, TCP, WSN cognitive radio.

Most of the commercial simulators are GUI (graphic user interface) driven while some network simulators are CLI (command user interface) driven. An important output of simulations is trace files. Trace files log every packet and event that occurred in simulation which will be used for analysis. Most network simulators use discrete event simulation, in which a list of pending "event" is stored and those events trigger future events such as the event of arrival of a packet at one node triggering the event of the arrival of that packet at the downstream node.

\subsection{Reasons for Wireless Simulators}

Wireless network simulation software provides modelling, simulation and analysis of wireless networks:

Modelling: Network simulation software allows modelling a network topology containing a wide variety of mobile devices that uses a multiple protocol. For example, you may need to model various types of cellular networks including GSM, CDMA, WiMAX or personal area network such as Bluetooth.

Simulation: Wireless network simulations can incorporate ground, wireless and satellite systems. These simulations allow one to study networking issues such as protocol interaction, congestion control and scalability. Fully realistic simulation reproduces the results of laboratory experiments: estimates the performance and behaviour of network use by end users.

Theoretical Analysis: Wireless network simulation software includes built in algorithms for analysing simulations. Often one can generate charts and graphs as well as export raw data to spreadsheets or math software for additional analysis. Some simulation software packages also have application programming interface for further customization [8].

\subsection{Types of Network Simulators}

There are different network simulators with different features. Some of the network simulators are: OPNET, NS2, NS3, NetSim, OMNeT++, REAL, J-Sim and QualNet. This software is available free or 
commercially over the network. In this section we will divide the open source network simulators based on their ease of availability. Table 1 is the comparison of different open-source network simulators.

\section{NS-3}

NS-3 is a discrete-event network simulator with a particular emphasis on network research and education. Users of NS-3 can construct simulations of computer networks using models of traffic generators, protocols and analyze or visualize the results. It is very helpful in research and education process due to its ability to obtain reproducible results: especially in wireless protocol design. NS-3 has the high degree of realism in the models including frameworks for real application and kernel code and integration of the tool with virtual machine environments and test beds. NS-3 has been in development since 2005 and has been making quarterly release since June 2008. It is designed as a set library that can combine together with other external software libraries. NS-3 is modular; several external animators and data analysis and virtualization tools can be used within NS-3. However, users should expect to work at a command line and with $\mathrm{C}++$ and/or python software development tools $[9,10]$.

\subsection{NS-3 Organizations}

The organization of the NS-3 code can be divided into three sections. The first section is the core and commonly used parts of the simulator like packets and the event scheduler. The second main section consists of the models that are used and needed for network simulation and lastly the third section are auxiliary helper functions to simplify setting up a simulation environment and tests to ensure correct functionality throughout version updates. The overview of software organization of NS-3 is illustrated in Fig. 1 below.

\subsection{OSI Model in NS-3}

NS-3 implements protocol up to layer 4 of the OSI model. On top of layer 4 , there are some applications that can be used to generate and receive traffic. Layer 4 features the TCP and UDP for IPv4 and IPv6 which is layer 3. NS-3 offers different routing algorithms like global routing for communication across the network which includes OLSR, AODV and DSDV. Layer 2 comprises of a higher and a lower MAC layer.

Table 1 Comparison of different open source simulators.

\begin{tabular}{|c|c|c|c|c|c|c|}
\hline $\begin{array}{l}\text { Name of } \\
\text { Simulator }\end{array}$ & Language & Platform & $\begin{array}{l}\text { Cost } \\
\text { \& License }\end{array}$ & Network Support Type & User Interface & API \\
\hline NS2 & $\mathrm{C}++, \mathrm{Otcl}$ & $\begin{array}{l}\text { Windows, } \\
\text { Linux }\end{array}$ & $\begin{array}{l}\text { Free, } \\
\text { Open Source }\end{array}$ & $\begin{array}{l}\text {-Wired Network, } \\
\text {-Wireless Ad-Hoc Mode, } \\
\text {-Wireless Managed Mode, } \\
\text {-Cannot simulate problems of the } \\
\text { bandwidth or the power } \\
\text { consumption in Wireless Sensor } \\
\text { Network }\end{array}$ & $\begin{array}{l}\text { Command Line } \\
\text { Interface }\end{array}$ & Pure Event Base \\
\hline NS3 & $\begin{array}{l}\text { C++, } \\
\text { Python }\end{array}$ & $\begin{array}{l}\text { Windows, } \\
\text { Linux, Mac OS }\end{array}$ & $\begin{array}{l}\text { Free, } \\
\text { GNU General } \\
\text { Public License }\end{array}$ & $\begin{array}{l}\text {-Wired Network } \\
\text {-Wireless Network } \\
\text {-Wireless Sensor Network }\end{array}$ & $\begin{array}{l}\text { Command Line } \\
\text { Interface }\end{array}$ & $\begin{array}{l}\text { Low-Level, } \\
\text { Users can mix and } \\
\text { match between the } \\
\text { simpler API }\end{array}$ \\
\hline OMNeT++ & $\mathrm{C}++$ & $\begin{array}{l}\text { Windows, } \\
\text { Unix-Based, } \\
\text { Mac OS X } 10.6 \\
\text { and } 10.7\end{array}$ & $\begin{array}{l}\text { Free, } \\
\text { Non-commercial } \\
\text { license, } \\
\text { Commercial } \\
\text { license }\end{array}$ & $\begin{array}{l}\text {-Wired Network } \\
\text {-Wireless Managed Mode }\end{array}$ & $\begin{array}{l}\text { Graphical User } \\
\text { Interface }\end{array}$ & Event Base \\
\hline J-Sim & $\begin{array}{l}\text { Java, } \\
\text { Tcl }\end{array}$ & $\begin{array}{l}\text { Truly platform } \\
\text { Independent }\end{array}$ & $\begin{array}{l}\text { Free, } \\
\text { Open Source }\end{array}$ & $\begin{array}{l}\text {-Wired Network, } \\
\text {-Wireless Network, } \\
\text {-Wireless Sensor Network, } \\
\text {-Radio Channels and power } \\
\text { consumptions }\end{array}$ & $\begin{array}{l}\text { GUI, } \\
\text { Command Line } \\
\text { Interface on } \\
\text { Linux }\end{array}$ & $\begin{array}{l}\text { Completely } \\
\text { process driven } \\
\text { including thread } \\
\text { synchronization }\end{array}$ \\
\hline
\end{tabular}




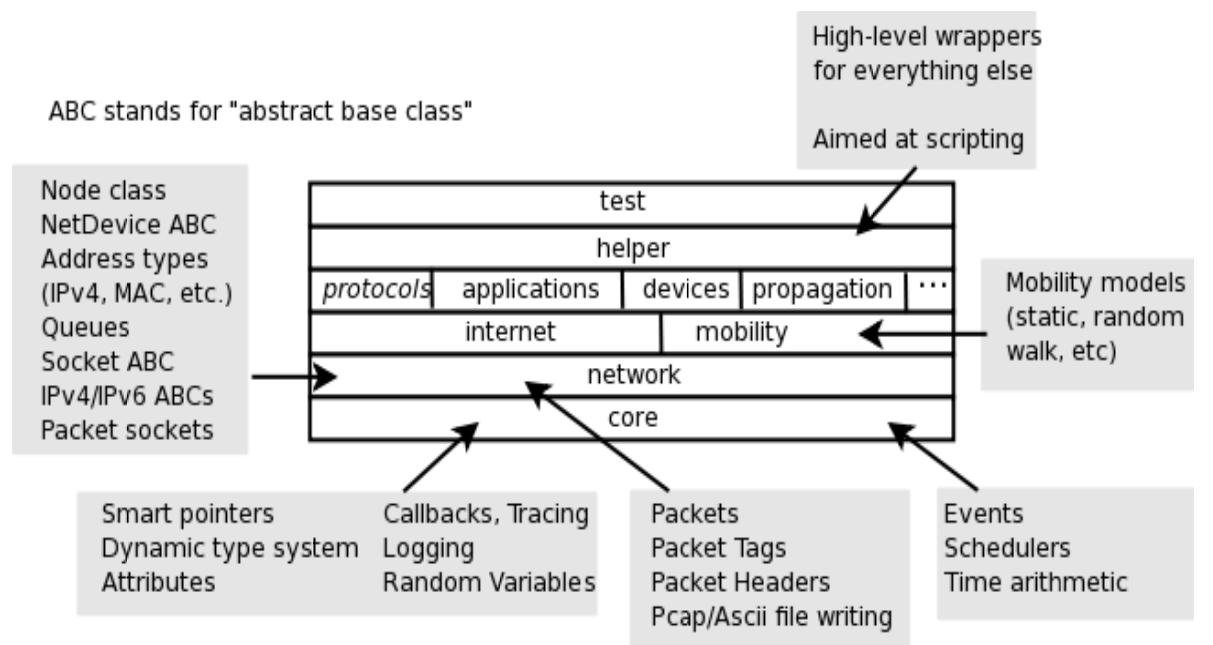

Fig. 1 NS3 simulator organization.

\begin{tabular}{|l|l|}
\hline \multicolumn{1}{|c|}{ OSI LAYERS } & Corresponding NS-3 Models \\
\hline Application & UDP echo,OnOffApplication, Sink \\
\hline Transport Layer & UDP, TCP \\
\hline Network Layer & IPv4/IPv6 Global (OSPF), Static Routing \\
\hline Link Layer & PointToPoint, CSMA, 802.11 \\
\hline Physical Layer & $\begin{array}{l}\text { Wired Channel (CSMA,PointToPoint) } \\
\text { Wireless Channels (802.11a, LTE) }\end{array}$ \\
\hline
\end{tabular}

Fig. 2 The layers in NS-3 according to the OSI layer model.

The higher MAC layer handles active probing as well as a packet queue, packet fragmentation and packet retransmission if needed. The lower MAC layer is responsible for data transmission to the physical layer and basic transaction like acknowledgment of received packets or RTS/CTS messages. The physical and channel layer are responsible for the general properties of the medium. Models exist both in wired or wireless communication. Fig. 2 is the diagram illustrating the NS-3 model layers.

\subsection{Modelling Networks in NS-3}

To simulate network scenarios, the simulator provides models of the components of the network. The following components are relevant for modelling networks.

Node: A node is the basic computing device abstraction. It stands for communication point, such as an end system or router. This is represented in $\mathrm{C}++$ by the class node which provides methods for managing the representations of computing devices in simulations. The nodes are interconnected by channels, which represent the different forms and media of data transmission.

Application: The application is the basic abstraction for user program that generates some activity to be simulated. It is represented in $\mathrm{C}++$ by class application. The application class provides methods for managing the representations of the version of user-level applications in simulations. 


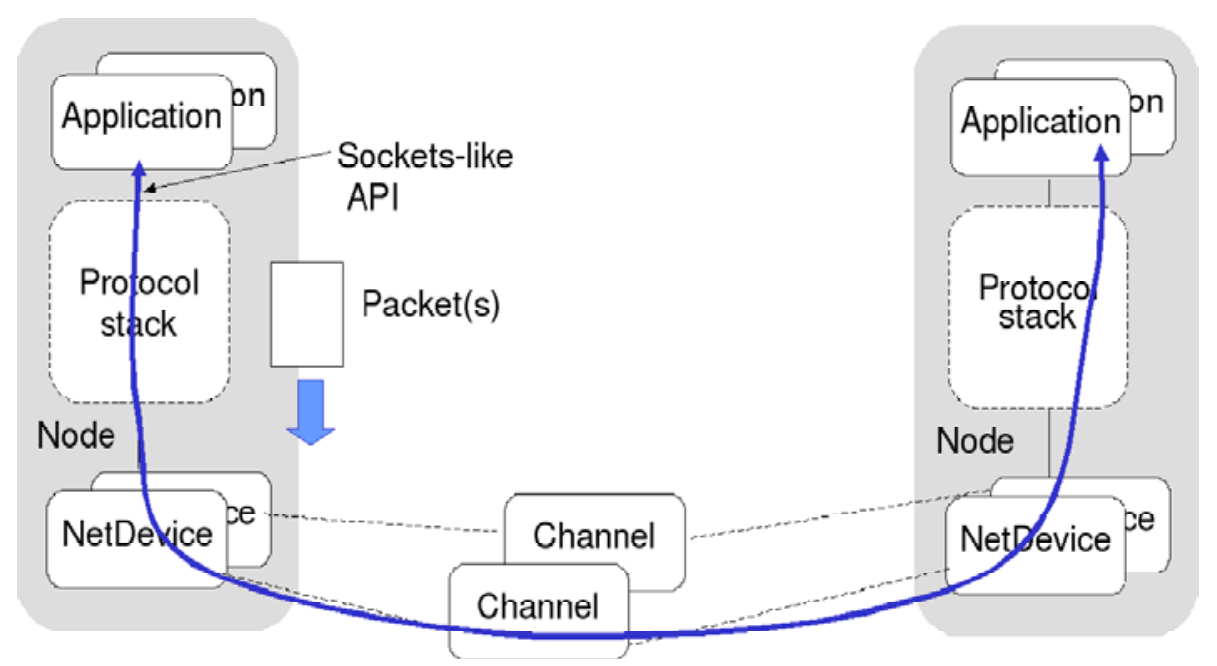

Fig. 3 NS-3 main components.

Channel: The channel class provides methods for managing communication sub network objects and connecting nodes to them.

Net Device: In NS-3, the net device covers both the software driver and simulated hardware. A net device is installed in a node in order to enable the node to communicate with other nodes in the simulation via channels.

Communications Protocols: This model the implementation of protocol descriptions found in various internets Request for Comments documents. These protocols objects are organized into protocol stack where each layer in the stack performs specific and limited function on network packets and then passes the packets to another layer for additional processing.

Network Packets: They are fundamental units of information exchange in computer network. A network packet would contain one or more protocol headers describing the information needed by the protocol implementation at the end points and various hops along the way. Further, the packets typically contain payload which represents the actual data being sent between end systems.

\section{Wi-Fi Network Simulation Setup and Results}

This section evaluates the implementation of Wi-Fi in NS-3. The experiment analyses throughput, delay (latency), jitter and packet loss. The topology of network illustrated in Fig. 4 below consists of one data source from station 1 which sends data to destinations through Access Point 1 in the BSS1 (basic service set) to the Access Point 2 in the second BSS2 to the destination node Station 4 within the same ESS (extended service set). This paper considers the infrastructure mode as shown. The network infrastructure constitutes four work stations and two access points for the purpose of analysing communication from one work station to another.

Fig. 5 is the simulation results and discussion obtained for throughput, delay and jitter which indicate an increase or a decrease in the respective parameters measured.

The throughput results in wireless networks under UDP showed that throughput easily reach $60 \mathrm{kbps}$ within the first two seconds and later decreases sharply then gentle. This is attributed to the introduction of background data traffic that competes with the data sent from the Station 1. The propagation of the data takes longer and thus the amount of packets that can be sent per second goes down because the sender is waiting for the receiver to acknowledge the reception which also takes longer.

The latency is low in this network under UDP protocol at initial stage then increases as time extends. 

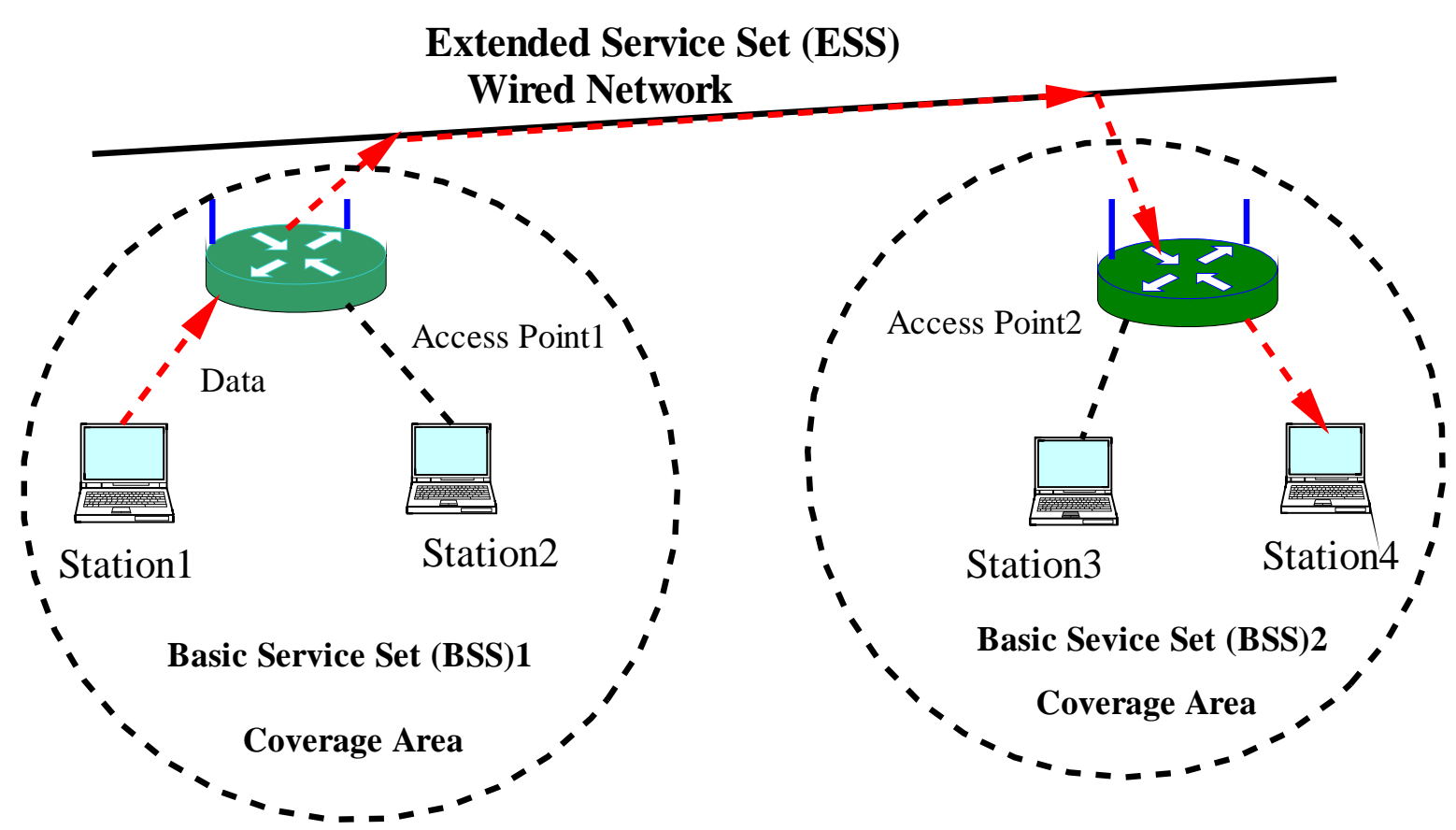

Fig. 4 The network topology for simulation.

\section{Throughput-Wi-Fi over UDP}

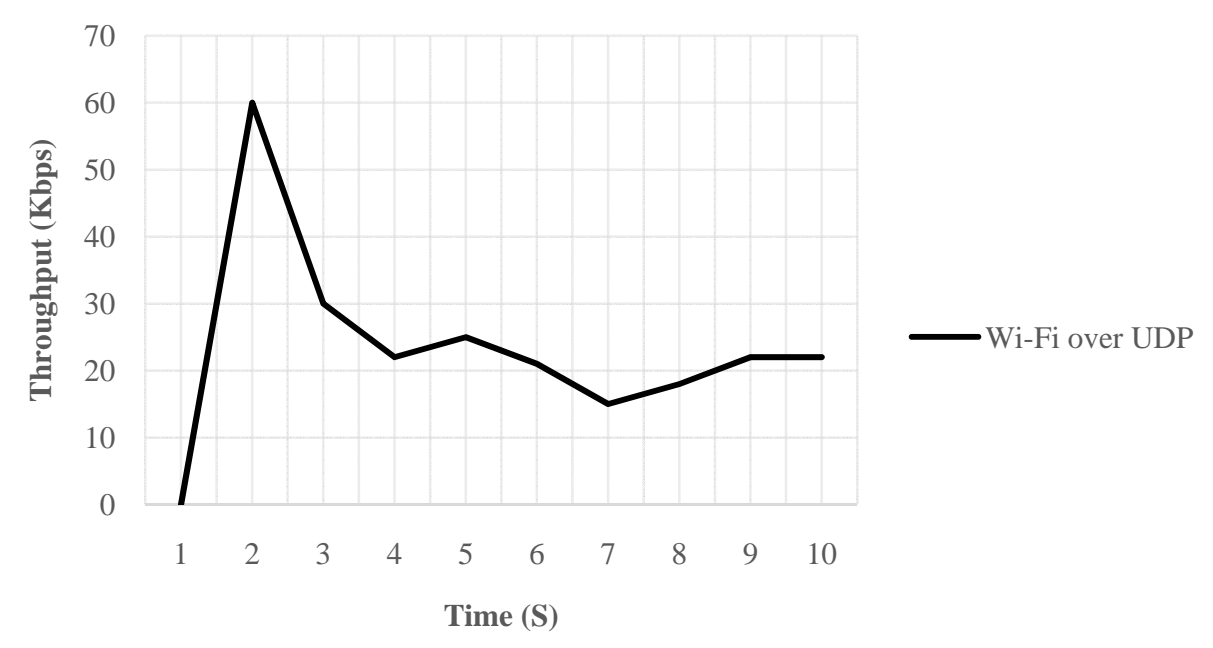

Fig. 5 Throughput-Wi-Fi over UDP.

However, the fluctuation of the latency is relatively higher in this wireless network topology in the presence of the other background traffic. If additional traffic for error detection and acknowledgment is added to the network the latency would be higher.

Latency has the correlation to the jitter; the difference in arrival time of the packets or data to the destination varies from time to time. Fig. 7 shows that jitter fluctuation increases for the wireless network. This may be as a result of wireless signals being prone to interference and signal instability.

The packet loss increases significantly because of 
increase in latency caused by queuing type of the duplex links, as there are three separate queues between sender and receiver, which results in undesired queue stacking characteristics resulting in significant amount of packet loss over long periods of times.

\section{Latency-Wi-Fi over UDP}

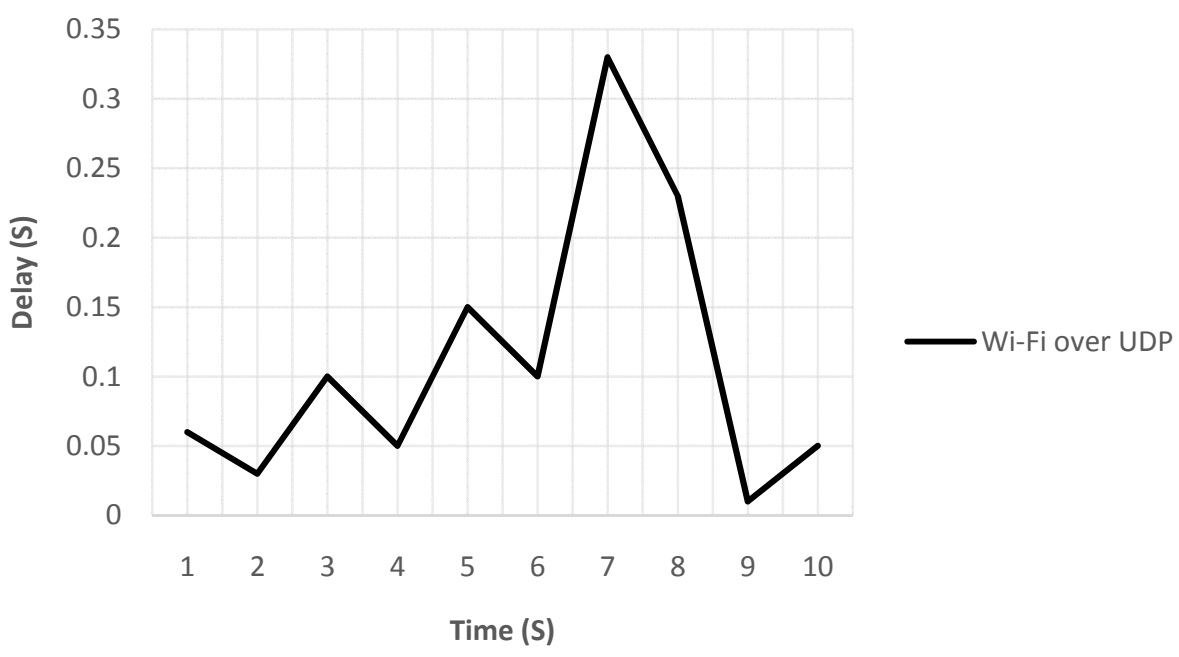

Fig. 6 Latency-Wi-Fi over UDP.

\section{Jitter-Wi-Fi Network vs Time}

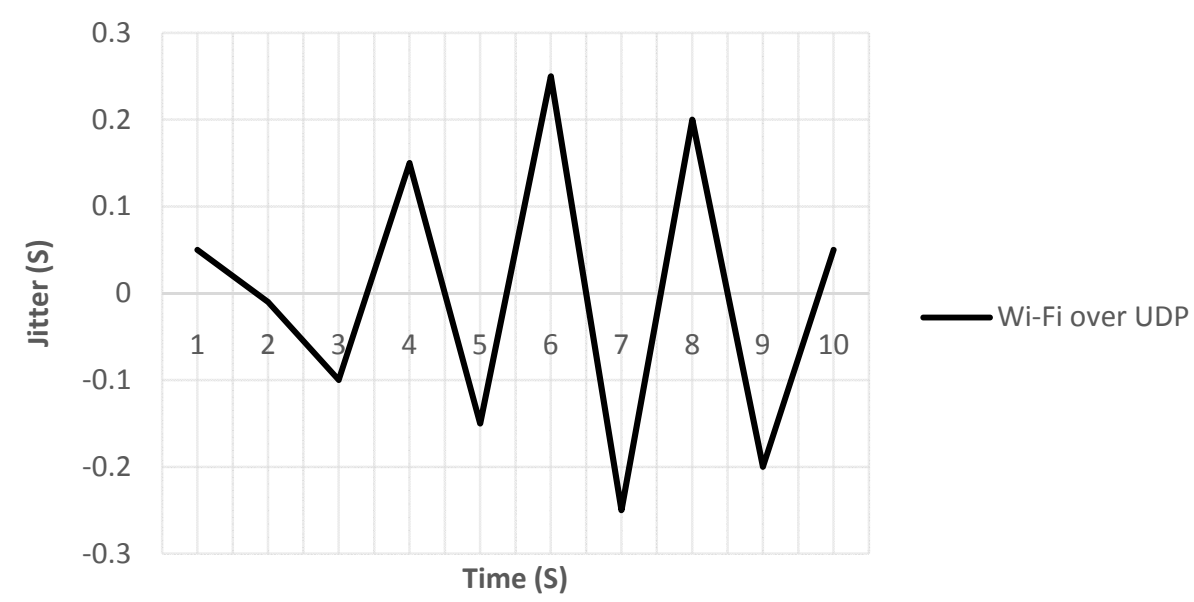

Fig. 7 Jitter-Wi-Fi over UDP. 


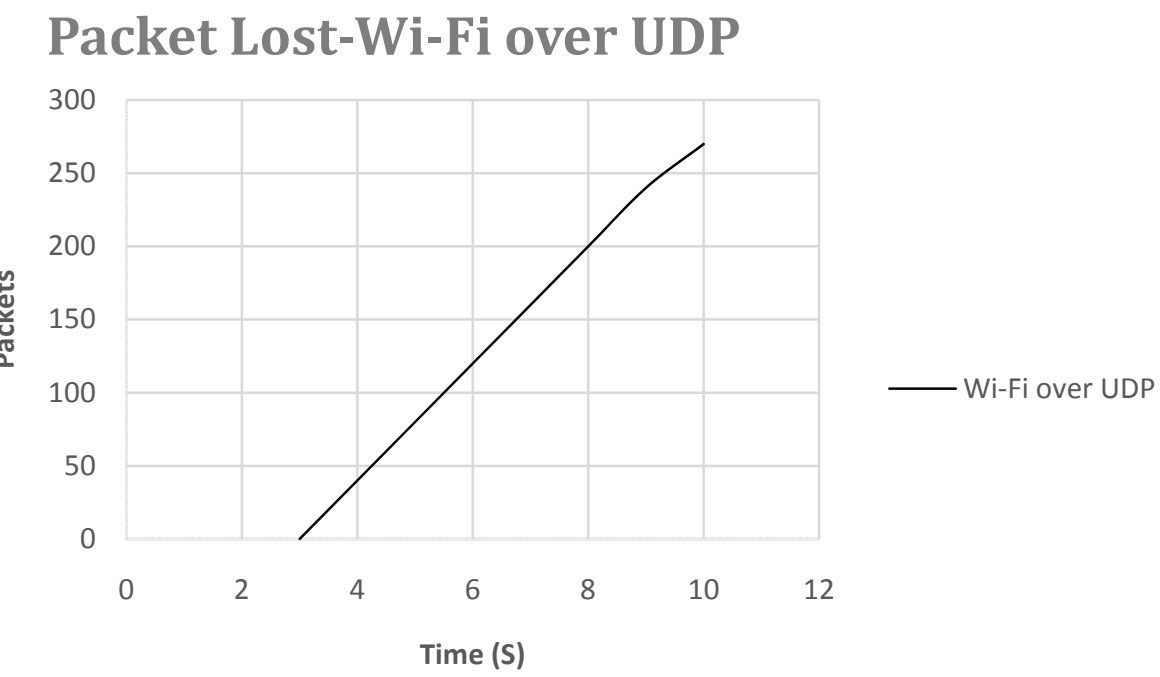

Fig. 8 Packet Loss-Wi-Fi over UDP.

\section{Conclusion}

In this paper, a Wi-Fi network is presented using NS-3 that provides tools to help implement networks and analysing it by plotting to graph. The paper presents the in-depth discussion NS3, highlighting its high degree of realism in the models including frameworks for real applications and kernel code and integration with virtual machine environments and test beds.

NS-3 is a useful educational tool for simulation of WLAN. By writing relatively simple simulation scripts, this paper has been able to evaluate the performance of Wi-Fi network using the parameters such as throughput, delay (latency), and jitter and packet loss. The implementation of Wi-Fi over NS-3 helps to improve, extend and modify existing protocols or replace them with one's own solutions.

\section{References}

[1] Perkins, C. E., and Royer, E. M. 2001. "Performance Valuation of Two On-demand Routing Protocol for Ad-Hoc Networks.” IEEE Personal Communication: 167-78.

[2] Types of Wireless Networks, [online]. Available: http://www.computernetworkingnotes.com/wireless-netw orking-on-cisco-route [Accessed: September 22, 2016].

[3] Al-Maashri, A., and Ould-Khaoua, M. 2006. "Performance Analysis of MANET Routing Protocol in the Presence of Self-similar Traffic.” In Proceeding of the 31st IEEE Conference on Local Computer Networks, 801-7.

[4] Nguyen, U. T., and Xiong, X. X. 2005. "Rate-Adaptive Multicast in Mobile Ad-Hoc Networks.” Department of Computer Science and Engineering York University Toronto, Canada M3J1P3.

[5] An introduction to wireless Networking (Part1)-802.11, Overview by Andrew Z. Tabona [Published on 20 May 2004/Last Updated on 20 May 2004].

[6] Wireless Fidelity-Wi-Fi, [online]. Available: https://www.ieee.org/about/technologies/emerging/wifi.p df [Accessed: September 22, 2016].

[7] Anand Nayyar et al. 2015. "A Comprehensive Review of Simulation Tools for Wireless Sensor Networks (WSNs).” Journal of Wireless Networking and Communications: 19-47.

[8] Wireless network simulators [online]. Available at: http://www.ehow.com/facts_7866977_wireless-network-s imulation-software.html.

[9] Wikipedia. "Network Simulation.” Available: http://en.wikipedia.org/wiki/Network_Simulation [Accessed: March 10, 2016].

[10] Network Simulator $3 . \quad$ Available: www.nsnam.org/docs/release/3.25/tutorial/singlehtml/ind ex.html [Accessed September 22, 2016]. 